\title{
Understanding of Controllable Optical Memory Using 1D Inp Based Photonic Structures at Three Communication Windows
}

\section{Rajesh Khanna M}

Vel Tech Multi Tech Dr Rangarajan Dr Sakunthala Engineering College

Karthikeyan Appathurai ( $\sim$ a.karthik1982@gmail.com )

Vel Tech Multi Tech Dr Rangarajan Dr Sakunthala Engineering College https://orcid.org/0000-00016290-6770

\section{Kuppusamy P G}

Siddhartha Institute of Engineering and Technology

\section{Prianka R R}

RMKCET: RMK College of Engineering and Technology

\section{Research Article}

Keywords: 1D optical memory, three communication windows, InP structure, Reflectance-transmittanceAbsorbance

Posted Date: January 3rd, 2022

DOI: https://doi.org/10.21203/rs.3.rs-1084087/v1

License: (1) This work is licensed under a Creative Commons Attribution 4.0 International License. Read Full License 


\section{Abstract}

The present research realises a controllable optical memory using one dimensional indium phosphate (InP) photonic structures at three optical communication windows $(850 \mathrm{~nm}, 1310 \mathrm{~nm}$ and $1550 \mathrm{~nm})$. The photonic structures comprise 21 layers of InP and air material. The memory applications are realised at both single and dual signals of the communication windows. The physics of the research deals with the materials property including the variation of the refractive indices with respect to the input signal. Similarly, mathematics of the works relies on the analysis of reflectance, transmittance and absorbance phenomena. Further, the light from visible spectrum acts as triggering signal to realise optical memory applications. Finally, it is revealed that $\ln P$ based photonic structures are suitable for controllable memory applications pertaining to the single wavelength $(850 \mathrm{~nm}, 1310 \mathrm{~nm}, 1550 \mathrm{~nm})$ or dual wavelengths ( $850 \mathrm{~nm}$ and $1310 \mathrm{~nm}, 1310 \mathrm{~nm}$ and $1550 \mathrm{~nm}, 1550 \mathrm{~nm}$ and $850 \mathrm{~nm}$ ).

\section{Introduction}

Research on photonics is not confined with the boundary wall of the single domain only. It plays a key role in driving different innovations pertaining to the new-fangled applications. The applications of photonics spread across various sectors including optical data communication, imaging, lightening, and displays. Apart from this, it also extends from manufacturing sector to heath care including security and safety [1]. Even though photonics have been used for different sectors, the present paper deals with the memory applications only. As far as literature review on the memory applications are concerned; reference [2] focuses on memory applications using two dimensional photonic crystal structures through the hysteresis features. Both plane wave expansion and finite difference time domain methods have employed in this work. In the reference [3], authors review state-of-the-art on integrated optical memories as well as random access memories with respect to their efficiency and performance. Similarly, in reference [4] nonlinear properties of the both 2D and 3D photonic crystal are thoroughly examined to realise photonic memory applications. Moreover reference [5] deals with the photonic crystal fiber to envisage memory application. In this research, no controllable memory was possible because maximum of two signals were considered. Further, in the reference [6], 151Eu3+:Y2SiO5-based quantum memories are demonstrated with the help of femto second-laser micromachining. Apart from this, optoelectronic material based resistive random access memory is discussed in the reference [7] using a three-layer vertical heterostructure of graphene and quantum dots. In the reference [8]; optoelectronics random access memory is realised using 2D dimensional heterostructures where the principle of the mechanism deals with neural network and image processing. One dimensional metamaterial [9] based photonic structure is used to understand optical memory applications where $650 \mathrm{~nm}$ and $850 \mathrm{~nm}$ act as excited and input signals respectively. Again, three dimensional photonic crystal structures is also employed in the reference [10] to understand the photonic memory applications for the signal of $1000 \mathrm{~nm}$ and $400 \mathrm{~nm}$ for input and excited signal respectively. Recently, a memory application is made using two dimensional structure of square, triangular and honeycomb crystal through the principle of absorption, reflection and transmission analysis [11]. In the reference [12], a non-volatile memory is designed with the help of phase 
change material $\left(\mathrm{Ge}_{2} \mathrm{Sb}_{2} \mathrm{Te}_{5}\right)$ based photonic structures. Further, in the reference [13] a non-volatile optical memory is designed with the help of silicon-oxide-nitride-oxide-silicon material, which is fitted in the photonic integrated circuit. A quantum computer memory is also discussed in the reference [14], where authors focus on the idea of the same. Lastly, news on the photonic memory is disclosed in the reference [15], where a notion of integrated optical memory and random access memory are presented. Even though different research have made for optical memory, the present research deals with one dimensional photonic structures for photonic memory application where InP is acted as background material. The present paper has a couple of advantages; such as

1. Proposed structure is $\operatorname{InP}$ based one dimensional crystal which is easy to fabricate.

2. It is controllable memory because the signal(s) can be emerging out depends on the excited signal

3. The proposed signals are belonged to the three optical communication windows $(850 \mathrm{~nm}, 1310 \mathrm{~nm}$ and $1550 \mathrm{~nm}$ )

\section{Structure And Mechanism}

The proposed structure is one dimensional photonic crystal that consists of 21 layers of indium phosphate (11 layers) and air (10 layers) material, which is shown in the figure 1(a);

In the figure 1; the thickness of the $\mathrm{InP}$ and air layer is taken of $1000 \mathrm{~nm}$ and $100 \mathrm{~nm}$ respectively. Here $\ln \mathrm{P}$ is chosen in odd potions where air is etched in the even position. Moreover the background material is InP where column material is taken as air. The reason for choosing such material, structure and dimension is that a controllable memory could be realised at this condition. Further the above structure is suitable for both first $(850 \mathrm{~nm})$, second $(1310 \mathrm{~nm})$ and third $(1550 \mathrm{~nm})$ optical communication windows. Further the working mechanism of the works relies on the different internal parameters of the structure such as refractive index of the background and column materials pertaining to the incident signal. Apart from this, it also depends on the thickness of material. Moreover, the operational mechanism can be understood from the figure 1(b) for realising optical memory applications.

Figure 1(b) gives an idea of an operational mechanism to understand the memory application with the help of one dimensional photonic structure. To understand the entire mechanism, we consider three steps as per following:

Step-1:

In the first step; three optical communication windows such as signals of $850 \mathrm{~nm}, 1310 \mathrm{~nm}$ and $1550 \mathrm{~nm}$ incident to the $\mathrm{InP}$ based photonic structure.

Step-2:

In the second step; when signals enter to the structure, there are three basic phenomena such as reflectance, transmittance and absorbance take place. Here the structure is designed in such way that it 
will neither reflect nor transmit any signals. It indicates the entire signals ( $850 \mathrm{~nm}, 110 \mathrm{~nm}$ and $1550 \mathrm{~nm}$ ) absorbed by this structure or stores all information, which is clearly shown in this diagram.

Step-3:

When external signal or excited signal is applied to the structure (which stores all the information), structure transmits the storing information or the absorbing signals. This is the basic principle of the memory. However the newness of the works is that the structure transmits a single signal and retains other two signals or transmits two signals and retains one signal. Such transmission of either single or double wavelengths depends on the excited signal, which is called as controllable.

\section{Why It Is Controllable Memory}

From step-3 (section-2), it is realised that the transmission of either single wavelength (850 nm or 1310 $\mathrm{nm}$ or $1550 \mathrm{~nm}$ ) or dual wavelengths $(850 \mathrm{~nm}$ and $1310 \mathrm{~nm}$ or $1310 \mathrm{~nm}$ and $1550 \mathrm{~nm}$ or $1550 \mathrm{~nm}$ and $850 \mathrm{~nm}$ ) depends on the excited signals or external signals. The same can be understood from the figure 2;

In the figure 2; it is observed that different excited signals have applied to the structure which contains information. For example; the signal $425 \mathrm{~nm}, 470 \mathrm{~nm}, 510 \mathrm{~nm}, 540 \mathrm{~nm}, 570 \mathrm{~nm}$ and $610 \mathrm{~nm}$ are applied to the structure then single or dual wavelengths have been transmitted from it. It indicates that the transmitted wavelength depends on the excited signal. That means the output or transmitted signal is controlled by excited wavelength. For example; if we transmit single wavelength then one has to excite by either $425 \mathrm{~nm}$ or $470 \mathrm{~nm}$ or $510 \mathrm{~nm}$ signal. It indicates that the signal, $425 \mathrm{~nm}, 470 \mathrm{~nm}$ and $510 \mathrm{~nm}$ excite and transmit the wavelength of $850 \mathrm{~nm}, 1310 \mathrm{~nm}$ and $1550 \mathrm{~nm}$ respectively. Similarly, one can realise the transmission of dual wavelengths with respect to the excited signals of $540 \mathrm{~nm}, 570 \mathrm{~nm}$ and $610 \mathrm{~nm}$ wavelength. To sum up; it is understood that the excited or trigging signals control the output wavelength which was stored in the structure. So the proposed structure is called as controllable memory because it stores the information and transmits it depending on our choice (triggering signals)

\section{Result And Interpretation}

Basically, the outcomes of the present research can be studied pertaining to the mechanism of the works which are reflected in the figure 1(b) and 2. From the step-1 and 2 of the figure 1(b), it is observed that signals of $850 \mathrm{~nm}, 1310 \mathrm{~nm}$ and $1550 \mathrm{~nm}$ incident it then zero reflectance and zero transmittance are found. The same can be understood from the figure 3(a) and 3(b) with respect to the reflectance and transmittance respectively.

Figure 3(a); Reflectance versus wavelength

In the figure 3; reflectance or transmittance (\%) is taken along vertical axis where wavelength of the signal is taken along horizontal axis. Though the wavelength is shown from $850 \mathrm{~nm}$ and $1550 \mathrm{~nm}$, three 
wavelengths such as $850 \mathrm{~nm}, 1310 \mathrm{~nm}$ and $1550 \mathrm{~nm}$ are focused because the effective refractive indices of the $\mathrm{InP}$ corresponding to these wavelengths taken during the simulation through plane wave expansion [16]. It is also found that zero reflectance ( from the figure 3(a)) and zero transmittance ( from the figure 3(b)) are associated with the structure. Then the absorbance of the signal is computed through numerical treatment [17] and its result is shown in the figure $3(\mathrm{c})$.

After the analysis of absorbance [ similar to the figure 3(a) and 3(b)] from the figure 3(c), the value of the same is found to be $100 \%$ at the signal of $850 \mathrm{~nm}, 1310 \mathrm{~nm}$ and $1550 \mathrm{~nm}$., which infers that whatever signal is applied to the structure, it absorbs the entire signals or information. These simulation and explanation validate the step-2 of the figure 1(b). Again, making the justification of the step-3, different signals have incident to the structure which is called as triggering or exciting wavelengths. An interestingly, different peculiar results have been observed. For example; when some spectrum of the visible signal is applied to the structure, it emanates the storing wavelength. The entire mechanism is discussed in the figure 2 where figure 4 and 5 validate the mechanism through the outcomes of the simulation.

Figure 4 and 5 represents the output results of the figure 2 pertaining to the transmission of single and dual wavelengths respectively. In this case, there is a certain assumption made with respect to the percentage of transmission of signal. For example; if the transmittance is more than $50 \%$, it is believed than signals are transmitted otherwise signals are not transmitted. Keeping the above presumption and analysing the figure 2 (a) and $4(\mathrm{a})$, the transmittance is $99 \%, 0 \%$ and $0 \%$ with respect to the signal of 850 $\mathrm{nm}, 1310 \mathrm{~nm}$ and $1550 \mathrm{~nm}$ respectively which infers that the wavelength $850 \mathrm{~nm}$ is only transmitted through the structure. So one can confirm from the above representation that if the signal of $425 \mathrm{~nm}$ is applied to the information containing structure then the signal $850 \mathrm{~nm}$ is transmitted where other two signals ( $1310 \mathrm{~nm}$ and $1550 \mathrm{~nm}$ ) would be retained by the structure. Even though the outcomes of the other results are shown for in the figure 4-5, the table-1 provides the complete information through which one can understand easily. 
Table 1

; Complete information to realise the controllable memory

\begin{tabular}{|c|c|c|c|c|c|c|c|c|}
\hline \multirow{2}{*}{$\begin{array}{l}\text { Excited } \\
\text { signal } \\
(\mathrm{nm})\end{array}$} & \multicolumn{3}{|c|}{$\begin{array}{l}\text { Transmittance }(\%) \text { at } \\
\text { communication windows }(\mathrm{nm})\end{array}$} & \multicolumn{3}{|c|}{ Analysis of result (\%) } & \multirow[t]{2}{*}{$\begin{array}{l}\text { Transmitted } \\
\text { signal (nm) }\end{array}$} & \multirow{2}{*}{$\begin{array}{l}\text { Retaining } \\
\text { signal } \\
\text { (nm) }\end{array}$} \\
\hline & 850 & 1310 & 1550 & 850 & 1310 & 1550 & & \\
\hline \multirow[t]{2}{*}{425} & 99 & 0 & 0 & $>50 \%$ & $<50 \%$ & $<50 \%$ & 850 & 1310 \\
\hline & & & & & & & & 1550 \\
\hline \multirow[t]{2}{*}{470} & 0.5 & 89.5 & 45 & $<50 \%$ & $>50 \%$ & $<50 \%$ & 1310 & 850 \\
\hline & & & & & & & & 1550 \\
\hline \multirow[t]{2}{*}{510} & 0 & 0 & 99 & $<50 \%$ & $<50 \%$ & $>50 \%$ & 1550 & 850 \\
\hline & & & & & & & & 1310 \\
\hline \multirow[t]{2}{*}{540} & 98 & 59 & 1 & $>50 \%$ & $>50 \%$ & $<50 \%$ & 850 & \\
\hline & & & & & & & 1310 & 1550 \\
\hline \multirow[t]{2}{*}{570} & 4 & 99.5 & 85 & $<50 \%$ & $>50 \%$ & $>50 \%$ & 1310 & 850 \\
\hline & & & & & & & 1550 & \\
\hline \multirow[t]{2}{*}{610} & 99.7 & 0 & 94 & $>50 \%$ & $<50 \%$ & $>50 \%$ & 850 & 1310 \\
\hline & & & & & & & 1550 & \\
\hline
\end{tabular}

In the Table 1; column 1 gives the information of the excited signal, column 2, 3,4 provides the transmittance values at three communication windows, where column $5,6,7$ indicates that whether transmittance is less than or more than $50 \%$. Similarly, column 8 and 9 shows transmitted and retaining signals respectively. The outcomes of the table-1 claimed that the triggering signal $425 \mathrm{~nm}, 470 \mathrm{~nm}$ and $510 \mathrm{~nm}$ transmits a single wavelength of $850 \mathrm{~nm}, 1310 \mathrm{~nm}$ and $1550 \mathrm{~nm}$ respectively. Similarly, the triggering signal of $540 \mathrm{~nm}, 570 \mathrm{~nm}$ and $610 \mathrm{~nm}$ transmits a dual wavelength of $(850 \mathrm{~nm}, 1310 \mathrm{~nm})$, $(1310 \mathrm{~nm}, 1550 \mathrm{~nm})$ and $(850 \mathrm{~nm}, 1550 \mathrm{~nm})$ respectively.

\section{Conclusion}

InP based one dimensional photonic structure is investigated in the present research to understand controllable memory where memory is realised through the transmittance of single or dual wavelengths. Entire mechanism is governed by the phenomenon of reflectance, transmittance and absorbance. The reflectance and transmittance are computed through plane wave expansion method where absorbance is examined by the analytical treatment. Finally output result affirms that the excited signal of $425 \mathrm{~nm}, 470$ $\mathrm{nm}$ and $510 \mathrm{~nm}$ transmits the wavelength of $850 \mathrm{~nm}, 1310 \mathrm{~nm}$ and $1550 \mathrm{~nm}$ respectively. Similarly, the 
signal of $540 \mathrm{~nm}, 570 \mathrm{~nm}$ and $610 \mathrm{~nm}$ transmits of $(850 \mathrm{~nm}, 1310 \mathrm{~nm}),(1310 \mathrm{~nm}, 1550 \mathrm{~nm})$ and $(850$ $\mathrm{nm}, 1550 \mathrm{~nm}$ ) respectively.

\section{References}

1. Chrostowski, J., Lampropoulos, G.A., Measures, R.M. (Eds.); Applications of Photonic Technology; XIV, 566; 1995; Springer, New York.

2. AlirezaGeravandMohammadDanaieSaeedMohammadi; All-optical photonic crystal memory cells based on cavities with a dual-argument hysteresis feature; Optics Communications; Volume 430, 1 January 2019, Pages 323-335

3. Theoni Alexoudi, George Theodore Kanellos ,Nikos Pleros; Optical RAM and integrated optical memories: a survey; Light: Science \& Applications volume 9, Article number: 91 (2020) ; 6058

4. Yong Zhang, Yan Sheng, Shining Zhu, Min Xiao, and Wieslaw Krolikowski; Nonlinear photonic crystals: from 2D to 3D ; Optica ; Vol. 8, Issue 3, pp. 372-381 ; (2021)

5. . G.Palai'B.Nayak ${ }^{a}$ Santosh KumarSahoo ${ }^{b}$ Soumya RanjanNayak ${ }^{c} S . K$.Tripathy; Metamaterial based photonic crystal fiber memory for optical computer; Optik; Volume 171, October 2018, Pages 393-396

6. Chao Liu, Zong-Quan Zhou, Tian-Xiang Zhu, Liang Zheng, Ming Jin, Xiao Liu, Pei-Yun Li, Jian-Yin Huang, Yu Ma, Tao Tu, Tian-Shu Yang, Chuan-Feng Li, and Guang-Can Guo; Reliable coherent optical memory based on a laser-written waveguide ; Optica; Vol. 7,Issue 2, pp. 192-197, (2020)

7. Siyu Zhou and Bo Peng, Non-volatile optical memory in vertical van der Waals heterostructures; 2020 J. Semicond. 41072906

8. Feichi Zhou, ${ }^{1}$ Jiewei Chen, ${ }^{1}$ Xiaoming Tao, ${ }^{2}$ Xinran Wang, ${ }^{3}$ and Yang Chai; 2 D Materials Based Optoelectronic Memory: Convergence of Electronic Memory and Optical Sensor, AAAS Research; Volume 2019 |Article ID 9490413;

9. I.S.Amiri,P.Yupapin ${ }^{\mathrm{a}}$ G.Palai; Realization of photonic memory using 1D optical metamaterial structure through absorption, reflection, and transmission analysis; Optik; Volume 185, May 2019, Pages 264268;

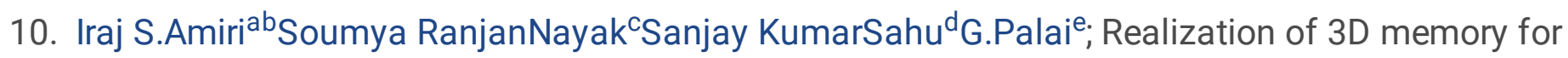
optical computer: A new paragon to future photonics; Optik; Volume 203, February 2020, 163914

11. Sangram Keshari Nayak; Subhankar Das;G. Palai; Realisation of photonic memory using S-T-a-H-p structures throughA-R-T Analysis;Microsystem Technologies (2021) 27:3141-3147;

12. Carlos Ríos; Nathan Youngblood, Zengguang Cheng, Manuel Le Gallo, Wolfram H. P. Pernice, C. David Wright, Abu Sebastian, Harish Bhaskaran; In-memory computing on a photonic platform Science Advances 15 Feb 2019;Vol. 5, no. 2, eaau5759

13. Irene Olivares; Jorge Parra; Pablo Sanchis; Non-Volatile Photonic Memory Based on a SAHAS Configuration; IEEE Photonics Journal ;Volume: 13 Issue: 2; (2021) ; page 1-5

14. Debanga Bhattasaly; Photonic Quantum Computer;(Optical Quantum Computer); International Journal of Engineering Research \& Technology (IJERT); ICRADL - 2021 Conference Proceedings 
Special Issue - 2021; 1-5, volume-5; issue-5

15. Alexoudi, T., Kanellos, G.T. \& Pleros, N.; A survey on optical memory and optical RAM technologies; July 21, 2020 ; https://phys.org/news/2020-07-survey-optical-memory-ram-technologies.html;

16. I.A. Sukhoivanov, I.V. Guryev, Physics and Practical Modeling: Photonic Crystals, Springer, Heidelberg, 2009.

MS Vijaya, G Rangarajan; Materials science; Tata McGraw-Hill, New Delhi, 2003

\section{Figures}

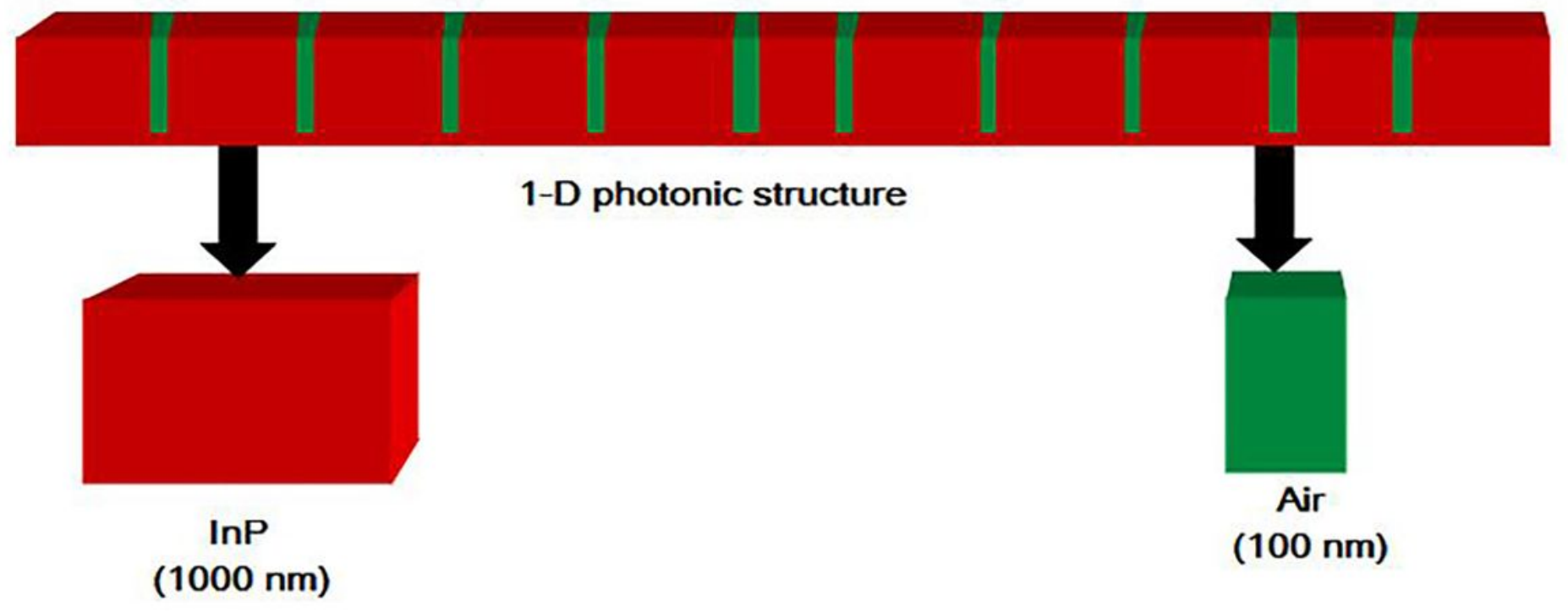

\section{Step-1}

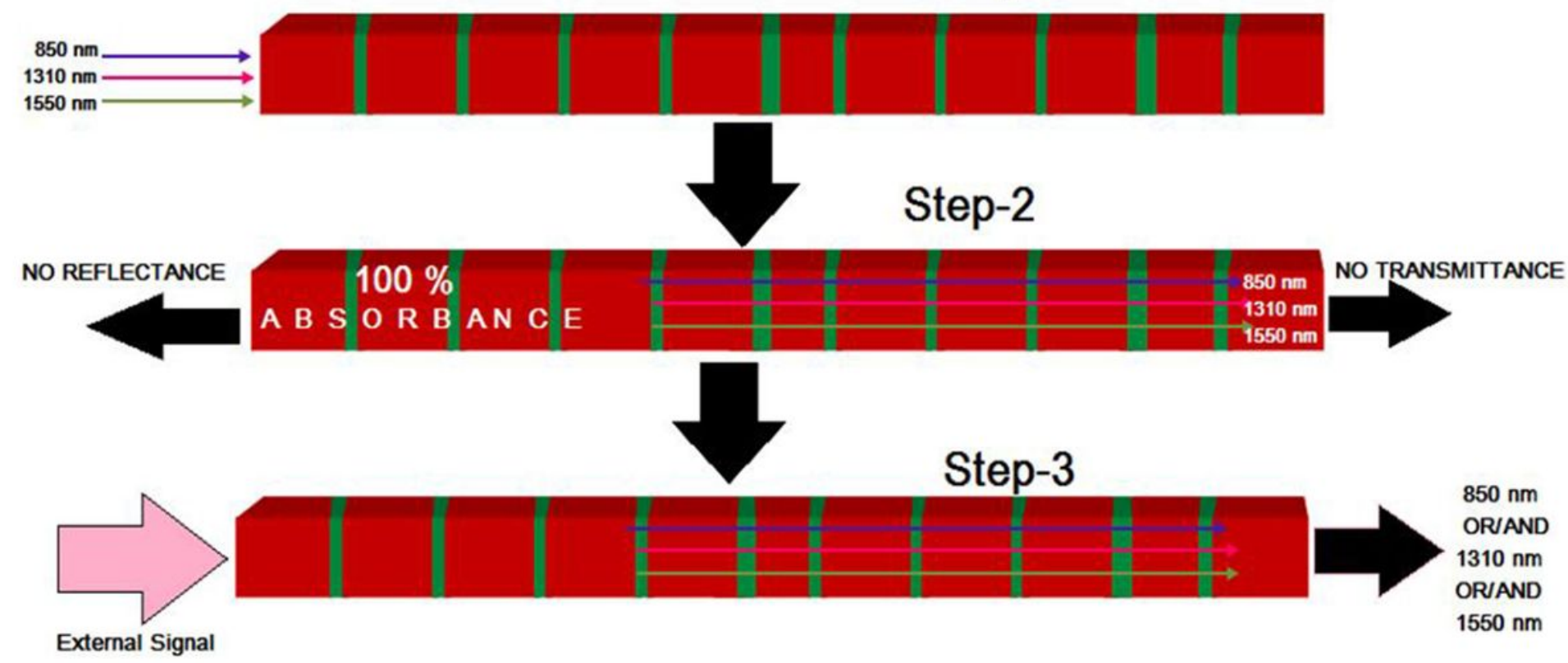


Figure 1

(a); Schematic diagram of 1D photonic structure(b); Operational mechanism to realise memory application

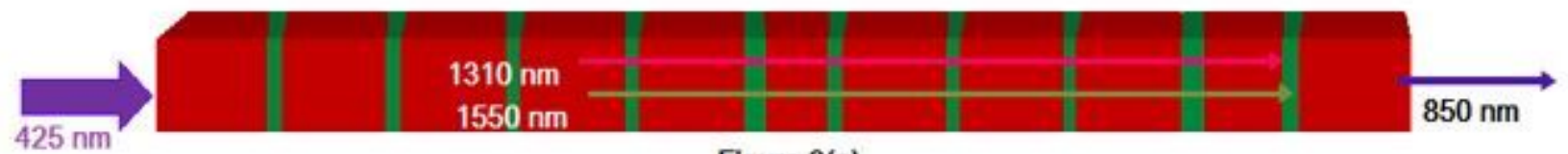

Figure 2(a)

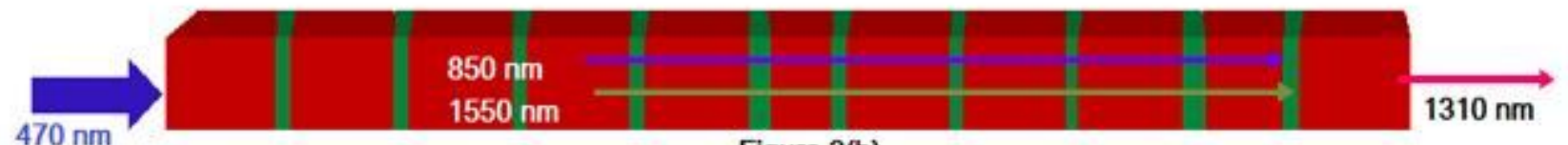

Figure 2(b)

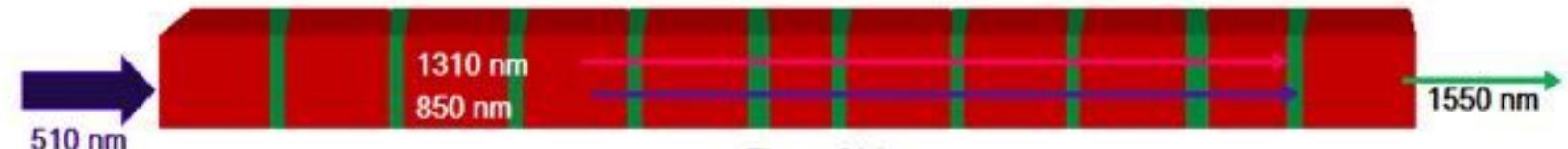

Figure 2(c)

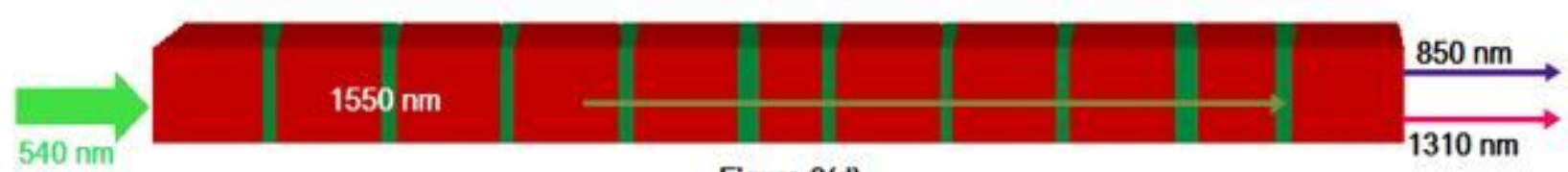

Figure 2(d)

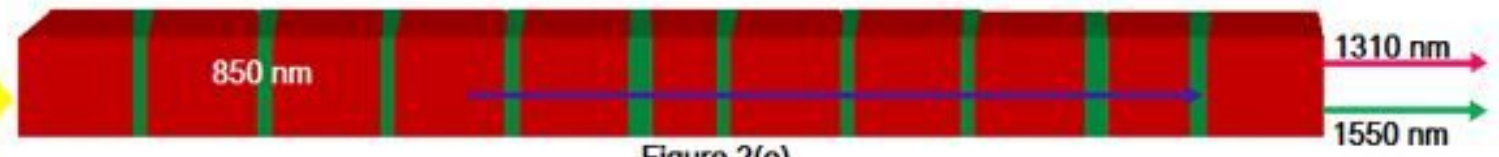

Figure 2(e)

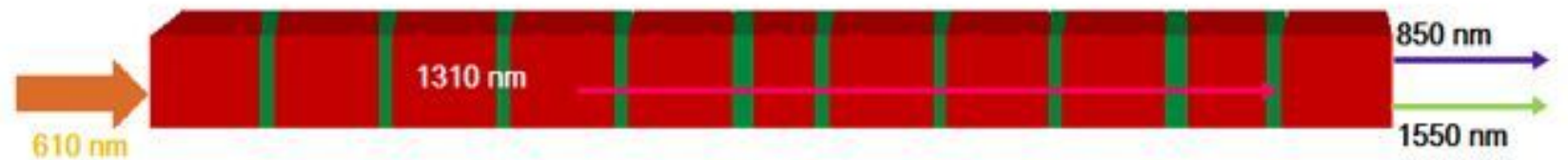

Figure 2(f)

Figure 2

excited signal and evacuated signal (a) $425 \mathrm{~nm}$ and $850 \mathrm{~nm}$ (b) $470 \mathrm{~nm}$ and $1310 \mathrm{~nm}$ (c) $510 \mathrm{~nm}$ and $1550 \mathrm{~nm}$ (d) $540 \mathrm{~nm}$ and $850 \mathrm{~nm}, 1310 \mathrm{~nm}$ (e) $570 \mathrm{~nm}$ and $1310 \mathrm{~nm}, 1550 \mathrm{~nm}$ (f) $610 \mathrm{~nm}$ and $850 \mathrm{~nm}$ , $1550 \mathrm{~nm}$. 

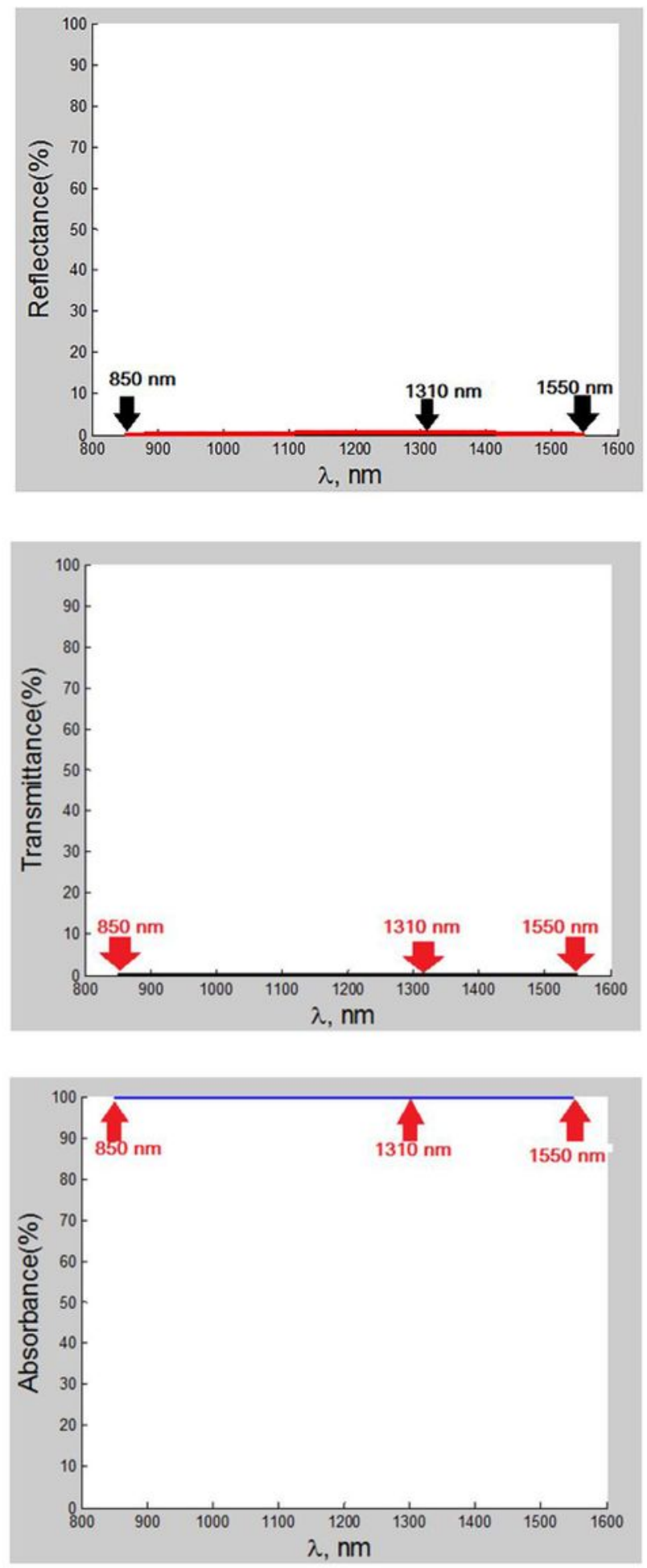

\section{Figure 3}

(a); Reflectance versus wavelength (b); Transmittance versus wavelength (c); Absorbance versus wavelength 

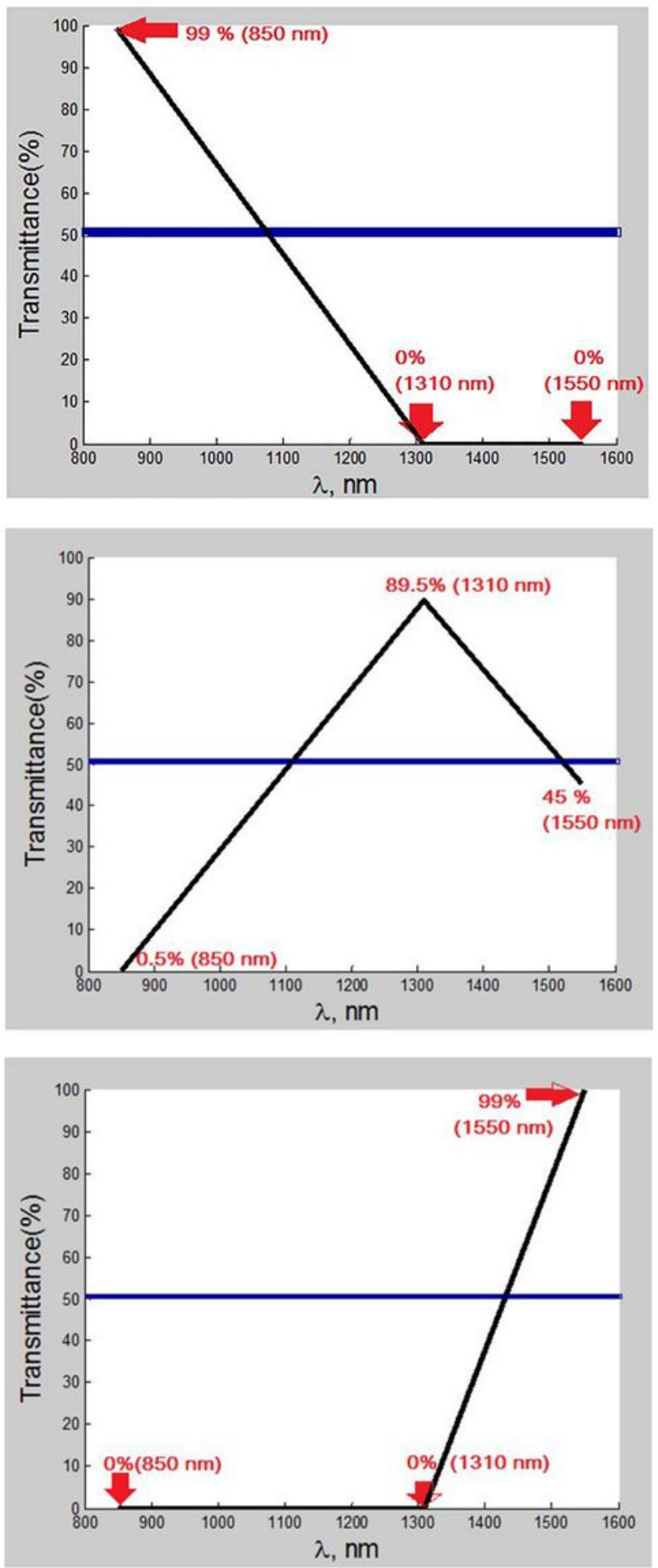

\section{Figure 4}

(a); Transmittance with respect to the allowance of $850 \mathrm{~nm}$ only (b); Transmittance with respect to the allowance of $1310 \mathrm{~nm}$ only (c); Transmittance with respect to the allowance of $1550 \mathrm{~nm}$ only 

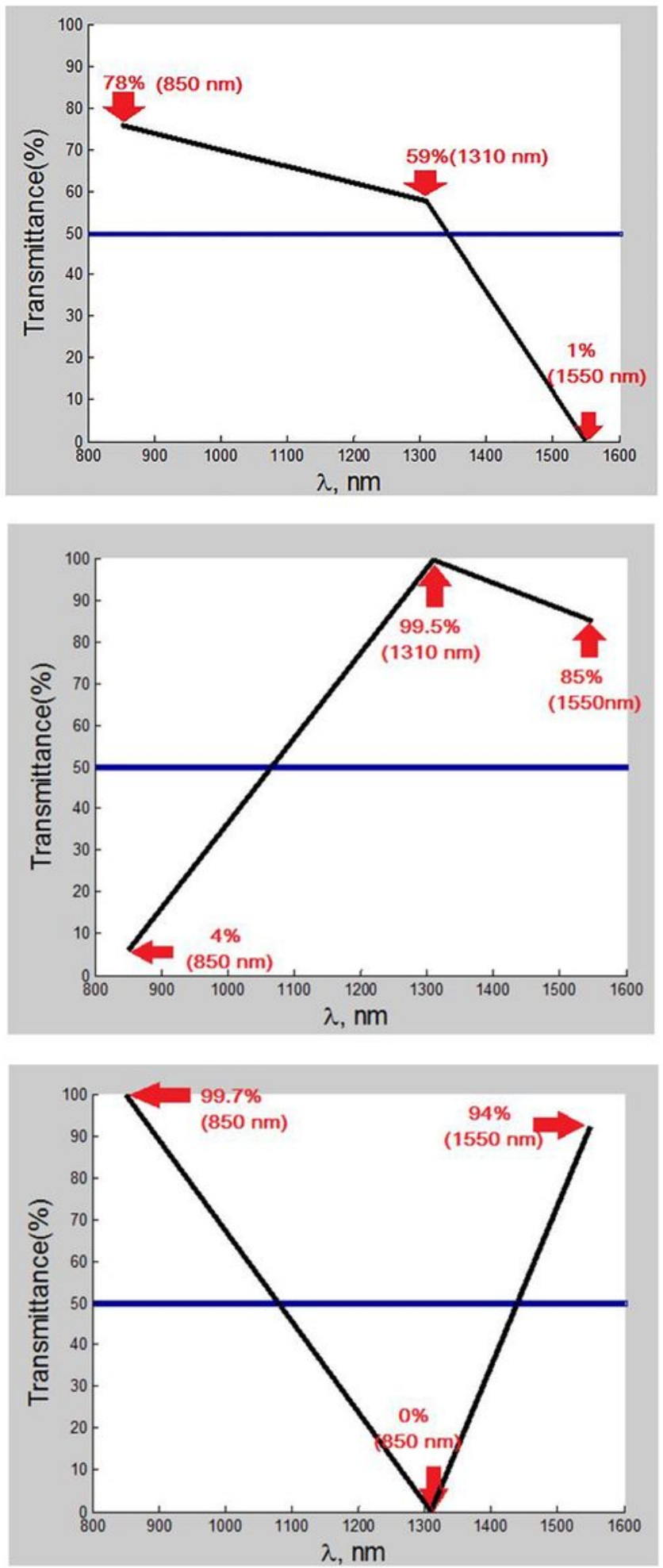

\section{Figure 5}

(a); Transmittance with respect to the allowance of $850 \mathrm{~nm}$ and $1310 \mathrm{~nm}$ (b); Transmittance with respect to the allowance of $1310 \mathrm{~nm}$ and $1550 \mathrm{~nm}$ (c); Transmittance with respect to the allowance of $850 \mathrm{~nm}$ and $1550 \mathrm{~nm}$ 